\title{
Expectativas en el consumo de alcohol en Bucaramanga, Colombia
}

\author{
María F. Ardila1,a, ó scar F. Herrán ${ }^{1,2, b .}$ \\ Expectancies towards alcohol \\ consumption in Bucaramanga, \\ Colombia
}

Background: Expectancies such as memories, motivations and emotions towards alcohol are an important predictor of alcohol consumption in the population. The Alcohol Expectancy Questionnaire (AEQ-III) can measure objectively these expectancies. Aim: To study which expectancies are associated to alcohol consumption in adults living in Bucaramanga, Colombia. Material and methods: The AEQ-III was applied to 601 adults (334 males) aged 18 to 60 years. A binomial regression was used to determine the expectancies that were associated with alcohol consumption after adjusting for social and demographic variables. Results: Eighty nine percent of the population consumes alcohol, without differences by age, gender, body mass index, educational or socioeconomic level. Positive expectancies related to disinhibition and feelings of power with a prevalence ratio (PR) of 1.33 (95\% confidence intervals of 1.06-1.67) and an age of less than 16 years when alcohol consumption started, with a PR of 1.15 (95\% confidence intervals of 1.08-1.23), were the only two explanatory variables for alcohol consumption. Conclusions: Alcohol comsuption is more associated to social context and group integration than with individual behaviors (Rev Méd Chile 2008; 136: 73-82).

(Key words: Alcohol drinking; Drinking behavior; Motivation)

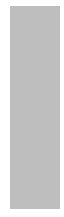

Recibido el 16 de enero, 2007. Aceptado el 24 de mayo, 2007.

${ }^{1}$ Observatorio Epidemiológico de Enfermedades Cardiovasculares, Centro de Investigaciones Epidemiológicas, Universidad Industrial de Santander, Bucaramanga, Colombia, ${ }^{2}$ Universidad Industrial de Santander, Escuela de Nutrición y Dietética.

${ }^{a}$ Enfermera. MSc. Epidemiología

${ }^{\mathrm{b}}$ Nutricionista Dietista. MSc. Epidemiología

$\mathrm{E}$ alcohol es la sustancia psicoactiva más consumida en el mundo, dependiendo de la cantidad y frecuencia consumida puede ser considerado como un nutriente, una toxina o una droga $^{1}$. Su consumo en el mundo occidental es

Correspondencia a: Óscar Fernando Herrán Falla. ND. MSc. Facultad de Salud. Tercer Piso. Universidad Industrial de Santander. Zona postal 3001. A.A. 2604. Bucaramanga. Colombia. Sur América. Telefax: (57-7) 6345781. E mail: herran28@intercable.net.co/herran@uis.edu.co aceptado y valorado socialmente. En Estados Unidos de Norteamérica, 51\% de la mujeres y $72 \%$ de los hombres lo consume; en adultos colombianos esta cifra alcanza a $90 \%{ }^{2}$.

El estudio del consumo de alcohol ha despertado gran interés en la salud pública durante las dos últimas décadas, pues se relaciona con el desarrollo de eventos epidémicos como los cardiovasculares ${ }^{3}$, cáncer ${ }^{4}$ y otros que están incrementándose y relacionados con la enfermedad social y mental $^{5}$; la violencia intrafamiliar ${ }^{6}$, la 
morbilidad y mortalidad por accidentes de vehículos automotores ${ }^{7}$; suicidio ${ }^{5}$, ausentismo laboral y escolar, problemas interpersonales ${ }^{8}$ y conductas sexuales peligrosas?. En la práctica clínica, el estudio de los determinantes del consumo de alcohol ha facilitado la implementación de estrategias de intervención como la del "consejero motivador" y la "conductual combinada", esta última suma a la consejería dirigida el uso de fármacos ${ }^{10-13}$.

El consumo se confunde frecuentemente con el abuso y la dependencia. El consumo es milenario, se asocia con la cultura y expresiones ceremoniales ${ }^{14}$; en cambio el abuso se asocia con un consumo patológico (excesivo o incontrolable), más deterioro o disfunción en el campo social u ocupacional. La dependencia, además de los criterios anteriores, debe llenar la evidencia de tolerancia o síndrome de abstinencia ${ }^{15}$.

En Bucaramanga, Colombia, estudios previos han mostrado que hombres y mujeres adultos, consumen alcohol, en promedio, cada siete días en niveles tóxicos ${ }^{16}$. Además, el riesgo de alcoholismo en esta población durante la última década ha sido mayor que el del país y ninguna variable biológica, sociodemográfica o dietaria ha sido asociada con el consumo de alcohol ${ }^{16}$. A pesar de la necesidad de estudiar la etiología del consumo de alcohol, en Bucaramanga y Colombia no existen investigaciones que se hayan aproximado a sus determinantes ${ }^{16,17}$.

La investigación en el campo de las expectativas hacia el alcohol (EA), se inició hace dos décadas y ha demostrado ser un predictor importante del consumo de alcohol en población adolescente y adulta ${ }^{18}$. Esta aproximación también llamada "de mecanismos de aprendizaje primitivos y habituales", toma aspectos de la memoria, de la motivación y las emociones de los sujetos y enfatiza sobre la capacidad de éstos para usar información genética o del ambiente para preparar la adaptación del comportamiento a circunstancias tardías ${ }^{19,20}$. Además, son un concepto psicosocial clave en la explicación del comportamiento hacia el consumo de alcohol y del abuso ${ }^{19,20}$. Las EA han sido medidas a través del Alcohol Expectancy Questionnaire (AEQ-III), éstas fueron redefinidas en 1980 por Brown y Goldman; "este constructo refleja la representación en la memoria de la información adquirida por un individuo alrededor de ciertos comportamientos dentro de contextos generales y específicos. Estas expectativas pueden ser adquiridas a través de experiencias directas, consecuencias observadas o de otras muchas formas en que los humanos adquieren el conocimiento. Esta memoria a su turno, estructura la percepción e interpretación del ambiente y por lo tanto determinan el comportamiento" 19,20 .

Las expectativas no sólo son mediadoras del efecto del alcohol sobre el comportamiento, el estado de ánimo y las emociones, sino que son factores de riesgo para la iniciación en el consumo, el mantenimiento del consumo, el consumo problemático y la dependencia ${ }^{19,20}$.

El objetivo de este estudio fue establecer cuáles expectativas hacia el alcohol están asociadas con su consumo en la población adulta de Bucaramanga, Colombia.

\section{Material y MÉTOdo}

En Bucaramanga, durante 2005-2006, se realizó un estudio cross-seccional, con los datos suministrados por 601 adultos residentes en el área urbana. Bucaramanga, es la ciudad más desarrollada de la región nororiental de Colombia, cuenta con 800.000 habitantes y su temperatura media es de $26^{\circ} \mathrm{C}$.

Tamaño de la muestra. El tamaño de la muestra fue calculado originalmente para obtener la validez factorial del AEQ-III (120 ítems x 5). Con un poder de $90 \%$ y un valor alfa de 0,05 , se requiere de 601 sujetos para observar diferencias de mínimo 8\% entre las EA, dado el hecho de consumir o no alcohol. Con 250 sujetos es posible con un poder de $92 \%$ y un alfa de 0,05, observar diferencias de mínimo $14 \%$ en las EA de acuerdo con el hecho de consumir alcohol ${ }^{21}$.

Selección de sujetos. Los que cumplieron los siguientes criterios fueron declarados elegibles: 1 . Entre 18 y 60 años. 2. Residir en la ciudad dos años o más. 3. No asistir a un grupo de apoyo como alcohólicos anónimos, y 4. Que manifestaran su deseo de participar suministrando voluntariamente la información. El nivel socioeconómico fue determinado por la oficina de planeación municipal. Los sujetos fueron seleccionados a 
través de muestreo multietápico para representar por género a la población. Primero, las manzanas de la ciudad se clasificaron en seis estratos socioeconómicos de acuerdo con la metodología de la Oficina de Planeación Municipal y veinte manzanas se seleccionaron aleatoriamente de cada estrato. Los mapas de estas 120 manzanas se actualizaron, las viviendas se numeraron consecutivamente y se eligieron al azar. En estas viviendas se hizo un censo de elegibles y finalmente se seleccionó un sujeto al azar por vivienda. Si la persona se rehusaba a participar, se elegía otra persona de la misma vivienda. Después de seleccionarlos, de informarlos sobre los objetivos, de garantizar la confidencialidad y de obtener un consentimiento escrito, se encuestaron. El proyecto y los procedimientos fueron aprobados por el Comité de Ética en investigación de salud de la Universidad Industrial de Santander.

Fuentes de información. Las fuentes de información fueron primarias. Dos encuestas fueron aplicadas mediante entrevista directa por una enfermera o nutricionista; un cuestionario sobre aspectos sociodemográficos que incluyó una pregunta sobre si había consumido alcohol durante el último año (sí o no) y el AEQ-III ${ }^{22}$. Los consumidores ceremoniales, es decir aquellos que sólo consumen hasta un trago o cerveza por ocasión, fueron considerados como no consumidores. AEQ-III es el cuestionario más usado cuando se estudian las expectativas hacia el consumo de alcohol, los encuestadores no necesitan de entrenamiento específico y sus 120 ítems pueden responderse entre 20 y $30 \mathrm{~min}$ por autorreporte o entrevista directa. AEQ-III evalúa las esperanzas (EA) que determinan el consumo de alcohol y mide el grado en que un individuo espera una variedad de efectos generales y específicos al consumir alcohol. La solución factorial del AEQ-III para Bucaramanga tiene una consistencia interna global de 0,85, un error de aproximación cuadrático medio (RMSEA) de 0,11 e índices de bondad de ajuste superiores a $0,80^{23}$. AEQ-III evalúa las EA hacia cuatro factores ${ }^{23} ; 1$. Incremento de la expresividad y la sexualidad, 2. Disminución de la tensión física, 3. Desinhibición y sentimientos de poder, y 4. Disminución de la tensión psicológica. Los detalles sobre la validación del AEQ-III y la solución factorial realizada para esta población fueron reportados previamente ${ }^{23}$. El peso corporal fue obtenido al momento de la encuesta mediante técnicas estandarizadas, utilizando básculas marca SOHENLE con sensibilidad de $100 \mathrm{~g}$.

Estadística. La descripción de las variables fue realizada con proporciones y promedios con sus intervalos de confianza del 95\% (IC; 95\%). También se reportó el promedio y la desviación estándar para las variables que dan origen a las EA. Para permitir la comparación, los puntajes alcanzados en cada una de las cuatro EA fueron escalas aditivas. Los estratos socioeconómicos 1 y 2, los más bajos en la escala de ingreso y desarrollo, se agruparon en el nivel socioeconómico 1 , los 3 y 4 en el nivel 2 y los estratos 5 y 6 en el nivel 3. Para establecer si entre grupos existían diferencias se utilizaron pruebas $\mathrm{t}$ de Student y de Chi-cuadrado. El análisis para determinar la mejor forma funcional de las variables, como la asociación entre el hecho de consumir o no alcohol (Variable dependiente), las EA y las variables sociodemográficas se realizó con regresión binomial ${ }^{24}$. Con base en esta regresión, se realizó un análisis multivariado para explicar el consumo de alcohol a través de las EA, ajustando por las variables sociodemográficas. La evaluación de confusión e interacción se hizo con la prueba de Mantel y Haenzel. Los errores estándar de todas las estimaciones se corrigieron por el efecto que tiene un muestreo multietápico como el aplicado; para ello se utilizó el método de aproximación de series lineales de Taylor (svy); la unidad primaria de muestreo fue el estrato socioeconómico (el primer paso del muestreo), para que las estimaciones de la varianza requirieran de pocas asunciones sobre la naturaleza de la muestra. Lo anterior permite cualquier cantidad de correlaciones en los sujetos dentro de la unidad primaria de muestreo. Así, los sujetos dentro de la unidad primaria de muestreo no tienen que ser independientes; esto es que pueden operar como cluster secundarios. Como resultado, la estimación de la varianza de medias y proporciones es más conservadora, tendiendo hacia valores más grandes. El efecto del diseño de la muestra sobre la varianza es conocido como el Deff y puede ser evaluado en términos relativos al calcular la razón entre la varianza lograda por el método de Taylor y la lograda al analizar los datos como si provinie- 
ran de un muestreo aleatorio simple 25,26 . Debido al diseño del estudio, los riesgos relativos a la exposición son equivalentes a razones de prevalencia (RP).

\section{Resultados}

Características de la población estudiada. Todos los sujetos respondieron las dos encuestas. Participaron entre 176 y 180 por cada nivel socioeconómico. El 55,6\% fueron hombres. La edad media fue de 34,7 años (IC; 33,8 a 35,6), sin diferencia por género. La edad media de inicio en el consumo de alcohol fue de 15,9 años (IC; 15,6 a 16,1), un año más tarde en las mujeres ( $\mathrm{p}=0,000)$. La edad media a la primera embriaguez fue de 17,6 años (IC; 17,3 a 17,9), dos años más tarde en las mujeres (p =0,000). El 4,7\% no terminó la educación básica primaria y 36,6\% terminó al menos once años de escolaridad. El 48,4\% tenía algún nivel de sobrepeso (índice de masa corporal mayor de 25). El 32\% y $57 \%$ reconocieron poco y moderado gusto por el consumo de alcohol respectivamente. El 88,7\% (IC; 85,4 a 91,9) de la población consumía alcohol, sin diferencias por edad, sexo, índice de masa corporal, la escolaridad o el nivel socioeconómico ( $p$
$>0,05$ ) (Tabla 1). La prevalencia de consumidores de alcohol, ajustada por el "factor de expansión" o ponderación del peso del cluster primario de muestreo fue de 90,5\% (IC; 87,2 a 93,8). Ninguna expectativa se asoció con la escolaridad o con el nivel socioeconómico ( $\mathrm{p}>0,05)$.

Expectativas y consumo de alcohol. Veintitrés variables que toman un valor de cero o uno, representan las cuatro EA. Un valor de uno significa una respuesta positiva. El promedio para estas variables osciló entre 0,64 y 0,74. El valor mínimo; 0,64, fue para la afirmación "soy mejor amante después de unos tragos" y el máximo; 0,74, para las afirmaciones "cuando estoy tomando me siento como yo quiero" y "cuando las mujeres toman, son más frescas con el sexo". Veinte de estas afirmaciones alcanzaron promedios mayores en los sujetos que reconocieron consumir alcohol ( $\mathrm{p}<0,05$ ) (Tabla 2). Consecuente con la teoría de las expectativas, los promedios en todas las EA fueron mayores en los consumidores de alcohol ( $\mathrm{p}<0,001)$. No se observaron diferencias por género (Tabla 3). Consistentemente, para las cuatro expectativas estudiadas fue mayor el puntaje en los sujetos que iniciaron el consumo antes de los 16 años de edad ( $\mathrm{x} \leq 0,05)$.

Tabla 1. C aracterísticas sociodemográficas

\begin{tabular}{|c|c|c|c|}
\hline Característica & Total [601] & Hombre [334] & Mujer [267] \\
\hline Edad (Años) & $34,7(33,8,35,6) *$ & $35,4(34,2,36,6)$ & $33,8(32,5,35,1)$ \\
\hline $\operatorname{IMC}\left(\mathrm{Kg} / \mathrm{m}^{2}\right)$ & $24,5(24,3,24,8)$ & $25,1(24,8,25,4)$ & $23,8(23,4,24,2)$ \\
\hline Años de educación & $12,8(12,5,13,1)$ & $12,8(12,3,13,2)$ & $12,8(12,3,13,2)$ \\
\hline Edad de inicio en el consumo & $15,9(15,6,16,1)$ & $15,1(14,8,15,5)$ & $16,8(16,3,17,2)$ \\
\hline Edad a la primera embriaguez & $17,6(17,3,17,9)$ & $16,9(16,5,17,2)$ & $18,5(18,1,18,9)$ \\
\hline \multicolumn{4}{|l|}{ Nivel socioeconómico** } \\
\hline Bajo & $200\{33,4\}^{* * *}$ & $119\{35,6\}$ & $81\{30,3\}$ \\
\hline Medio & $200\{33,3\}$ & $97\{29,0\}$ & $103\{38,6\}$ \\
\hline Alto & $200\{33,3\}$ & $118\{35,4\}$ & $83\{31,1\}$ \\
\hline \multicolumn{4}{|l|}{ ¿Enfermedad actual?****** } \\
\hline Sí & $24\{4,0\}$ & $13\{3,9\}$ & $11\{4,1\}$ \\
\hline
\end{tabular}

[n]. *Promedio e intervalo de confianza al 95\%. * Con base en la oficina de planeación municipal, el nivel bajo se corresponde con los estratos socioeconómicos 1 y 2 , el medio con los estratos 3 y 4 y el alto con los estratos 5 y $6 .{ }^{* * *}$ Frecuencia $\{\%\}$. ****Diagnosticada por un médico y que haya modificado la ingesta dietaria o de alcohol en el último año. 
- EXPECTATIVAS EN CONSUMO DE ALCOHOL EN BU CARAMANGA, COLOMBIA - M Ardila et al

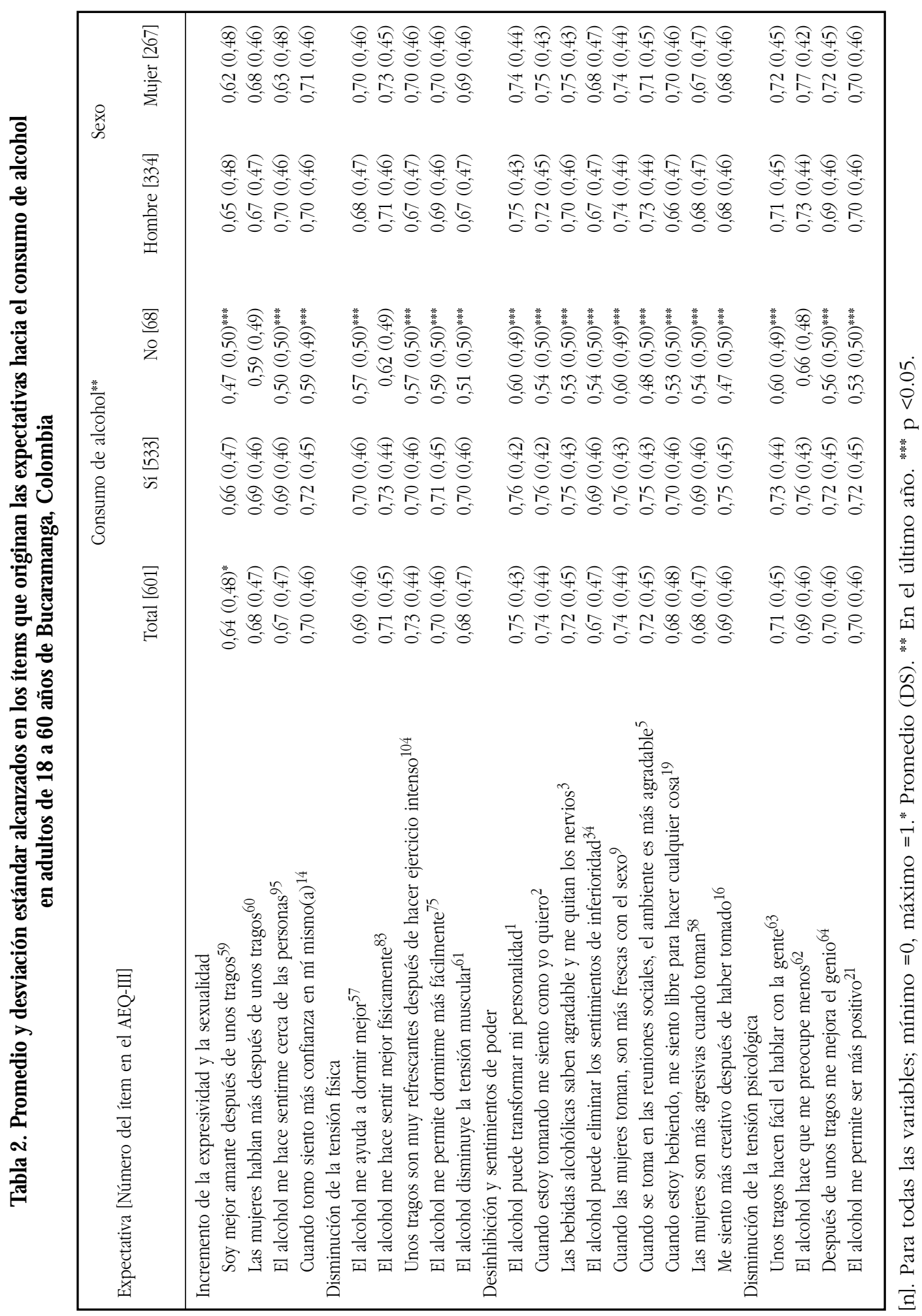


Tabla 3. Puntajes alcanzados en las expectativas hacia el consumo de alcohol en adultos de 18 a 60 años en Bucaramanga, Colombia.

\begin{tabular}{|c|c|c|c|c|c|c|}
\hline \multirow[b]{2}{*}{ Expectativa } & \multirow{2}{*}{ Rango } & & \multicolumn{2}{|c|}{ Consumo de alcohol** } & \multicolumn{2}{|c|}{ Sexo } \\
\hline & & [601] & $\begin{array}{r}\text { Sí } \\
{[533]}\end{array}$ & $\begin{array}{l}\text { No } \\
{[68]}\end{array}$ & $\begin{array}{c}\text { Hombre } \\
\text { [334] }\end{array}$ & $\begin{array}{l}\text { Mujer } \\
{[267]}\end{array}$ \\
\hline Incremento de la expresividad y la sexualidad & $0-4$ & $\begin{array}{r}2,69 * \\
(2,58,2,80) *\end{array}$ & $\begin{array}{r}2,76 \\
(2,64,2,89)\end{array}$ & $\begin{array}{r}2,15 \\
(1,81,2,45)\end{array}$ & $\begin{array}{r}2,72 \\
(2,57,2,88)\end{array}$ & $\begin{array}{r}2,65 \\
(2,45,2,82)\end{array}$ \\
\hline Disminución de la tensión física & $0-5$ & $\begin{array}{r}3,46 \\
(3,33,3,59)\end{array}$ & $\begin{array}{r}3,54 \\
(3,40,3,68)\end{array}$ & $\begin{array}{r}2,87 \\
(2,44,3,29)\end{array}$ & $\begin{array}{r}3,42 \\
(3,24,3,59)\end{array}$ & $\begin{array}{r}3,52 \\
(3,32,3,72)\end{array}$ \\
\hline Desinhibición y sentimientos de poder & $0-9$ & $\begin{array}{r}6,38 \\
(6,17,6,59)\end{array}$ & $\begin{array}{r}6,57 \\
(6,36,6,79)\end{array}$ & $\begin{array}{r}4,85 \\
(4,13,5,57)\end{array}$ & $\begin{array}{r}6,34 \\
(6,05,6,63)\end{array}$ & $\begin{array}{r}6,43 \\
(6,11,6,75)\end{array}$ \\
\hline Disminución de la tensión psicológica & $0-4$ & $\begin{array}{r}2,81 \\
(2,70,2,92)\end{array}$ & $\begin{array}{r}2,89 \\
(2,77,3,01)\end{array}$ & $\begin{array}{r}2,19 \\
(1,81,2,57)\end{array}$ & $\begin{array}{r}2,76 \\
(2,61,2,92)\end{array}$ & $\begin{array}{r}2,87 \\
(2,70,3,04)\end{array}$ \\
\hline
\end{tabular}

[n]. *Promedio e intervalo de confianza al 95\%. **En el último año, $\mathrm{p}<0,001$ para todas.

La estimación de RP crudas, mostró como la edad de inicio en el consumo de alcohol, la de la primera embriaguez y las cuatro EA estudiadas, son las únicas variables asociadas al hecho de consumir alcohol. Iniciar el consumo antes de los 16 años de edad, incrementa la probabilidad de ser un consumidor en $17 \%$ (IC; 9 a 24 ). De la misma forma, embriagarse antes de los 16 años aumenta la probabilidad de consumir alcohol en 11\% (IC; 6 a 17). Después de explorar diferentes puntos de corte para determinar su mejor forma funcional en un modelo binomial, las EA fueron llevadas a escalas dicotómicas, los puntos de corte seleccionados para todas están por debajo del percentil 25 de la distribución. Alcanzar un puntaje mayor de dos en las EA "incremento de la expresividad y la sexualidad" y "desinhibición y sentimientos de poder", aumenta la probabilidad para ser consumidor en 11\% (IC; 4 a 18) y $37 \%$ (IC; 9 a 73), respectivamente. Un puntaje mayor de uno en las EA "disminución de la tensión física" y "disminución de la tensión psicológica", aumenta la probabilidad de consumir alcohol en $14 \%$ (IC; 3 a 26) y $12 \%$ (IC; 3 a 22), respectivamente (Tabla 4).

Después de corregir la varianza por el diseño de la muestra y de ajustar las RP en un modelo binomial, sólo dos variables explican el hecho de consumir alcohol, las expectativas positivas relacionadas con la "desinhibición y los sentimientos de poder"; RP 1,33 (IC; 1,06 a 1,67) y la edad de inicio en el consumo, menor o igual a 16 años; RP 1,15 (IC; 1,08 a 1,23) (Tabla 5). No se evidenció confusión o interacción entre el hecho de consumir alcohol, las EA, el sexo, la edad, la edad de inicio en el consumo o de la primera embriaguez.

\section{DisCUSIÓN}

La proporción de hombres y mujeres estudiados, como la de sujetos sin educación básica primaria, se correspondió con la de la población general ${ }^{27}$. Además, la edad de inicio en el consumo de alcohol, la de la primera embriaguez y la proporción de consumidores, fueron similares a las reportadas en un estudio previo en la misma población ${ }^{16}$. A pesar de tener $55 \%$ de hombres en la muestra, no hubo evidencia de sesgo de selección o de participación diferencial. Todo lo anterior permite suponer que la muestra representó a la población de adultos satisfactoriamente.

Estudios previos han mostrado que las expectativas positivas hacia el consumo de alcohol están fuertemente asociadas con la cultura. En los adolescentes y adultos jóvenes las EA se reafirman o desechan en la medida en que el consumo incrementa ${ }^{9,18-20,22}$. Lo anterior presupone una diferenciación en las expectativas con la edad. En este estudio no se encontró evidencia de puntua- 
Tabla 4. Proporción de consumidores de alcohol y razones de prevalencia (RP) para algunas características sociodemográficas y las expectativas hacia el consumo de alcohol en adultos de 18 a 60 años en Bucaramanga, C olombia.

\begin{tabular}{|c|c|c|c|c|}
\hline \multirow[b]{2}{*}{ Variable } & \multicolumn{4}{|c|}{ Consumo de Alcohol } \\
\hline & $\begin{array}{c}\text { Sí } \\
\mathrm{n}(\%)\end{array}$ & $\begin{array}{c}\text { No } \\
\text { n }(\%)\end{array}$ & RP (95\% IC) & Valor $\mathrm{p}$ \\
\hline \multicolumn{5}{|l|}{ Edad (Años) } \\
\hline $15-24$ & $98(89,1)$ & $12(10,9)$ & 1,00 & \\
\hline $25-39$ & $273(89,2)$ & $33(10,8)$ & $1,00(0,93,1,08)$ & 0,971 \\
\hline $40-60$ & $162(87,6)$ & $23(12,4)$ & $0,98(0,90,1,08)$ & 0,691 \\
\hline \multicolumn{5}{|l|}{ Sexo } \\
\hline Mujer & $230(86,1)$ & $37(13,9)$ & 1,00 & \\
\hline Hombre & $303(90,7)$ & $31(9,3)$ & $1,05(0,99,1,11)$ & 0,086 \\
\hline \multicolumn{5}{|l|}{$\operatorname{IMC}\left(\mathrm{Kg} / \mathrm{m}^{2}\right)$} \\
\hline$\leq 25$ & $273(88,1)$ & $37(11,9)$ & 1,00 & \\
\hline$>25$ & $260(89,3)$ & $31(10,7)$ & $1,01(0,96,1,07)$ & 0,619 \\
\hline \multicolumn{5}{|l|}{ Escolaridad (años) } \\
\hline$\leq 5$ & $25(89,3)$ & $3(10,7)$ & 1,00 & \\
\hline 6 a 11 & $195(88,6)$ & $25(11,4)$ & $0,99(0,86,1,14)$ & 0,917 \\
\hline$>12$ & $313(88,7)$ & $40(11,3)$ & $0,99(0,87,1,13)$ & 0,919 \\
\hline \multicolumn{5}{|l|}{ Nivel socioeconómico* } \\
\hline Bajo & $180(90,0)$ & $20(10,0)$ & 1,00 & \\
\hline Medio & $177(88,5)$ & $23(11,5)$ & $0,98(0,92,1,05)$ & 0,628 \\
\hline Alto & $176(87,6)$ & $25(12,4)$ & $0,97(0,91,1,04)$ & 0,440 \\
\hline \multicolumn{5}{|l|}{ ¿Alguna dieta en el último año? } \\
\hline Sí & $84(82,3)$ & $18(17,7)$ & & 1,00 \\
\hline No & $449(90,0)$ & $50(10,0)$ & $1,09(0,99,1,20)$ & 0,066 \\
\hline \multicolumn{5}{|l|}{ ¿Enfermedad actual?*** } \\
\hline Sí & $19(79,2)$ & $5(20,8)$ & 1,00 & \\
\hline No & $514(89,1)$ & $63(10,9)$ & $1,12(0,91,1,38)$ & 0,264 \\
\hline \multicolumn{5}{|l|}{ Edad de inicio en el consumo (años) } \\
\hline$>16$ & $208(80,9)$ & $49(10,1)$ & 1,00 & \\
\hline$\leq 16$ & $325(94,5)$ & $19(5,5)$ & $1,17(1,09,1,24)$ & 0,000 \\
\hline \multicolumn{5}{|l|}{ Edad de la primera embriaguez (años) } \\
\hline$>16$ & $338(85,3)$ & $58(14,7)$ & 1,00 & \\
\hline$\leq 16$ & $195(95,1)$ & $10(4,9)$ & $1,11(1,06,1,17)$ & 0,000 \\
\hline \multicolumn{5}{|c|}{ Incremento de la expresividad y la sexualidad (puntaje) } \\
\hline$\leq 2$ & $191(83,0)$ & $39(17,0)$ & 1,00 & \\
\hline$>2$ & $342(92,2)$ & $29(7,8)$ & $1,11(1,04,1,18)$ & 0,002 \\
\hline \multicolumn{5}{|l|}{ Disminución de la tensión física (Puntaje) } \\
\hline$\leq 1$ & $81(79,4)$ & $21(20,6)$ & 1,00 & \\
\hline$>1$ & $452(90,6)$ & $47(9,4)$ & $1,14(1,03,1,26)$ & 0,012 \\
\hline \multicolumn{5}{|c|}{ Desinhibición y sentimientos de poder (puntaje) } \\
\hline$\leq 2$ & $25(65,8)$ & $13(34,2)$ & 1,00 & \\
\hline$>2$ & $508(90,2)$ & $55(9,8)$ & $1,37(1,09,1,73)$ & 0,007 \\
\hline Disminución de la tensión psicológica & (puntaje) & & & \\
\hline$\leq 1$ & $112(81,2)$ & $27(18,2)$ & 1,00 & \\
\hline$>1$ & $416(90,0)$ & $41(9,0)$ & $1,12(1,03,1,22)$ & 0,008 \\
\hline
\end{tabular}

* Con base en la oficina de planeación municipal, el nivel bajo se corresponde con los estratos socioeconómicos 1 y 2 , el medio con los estratos 3 y 4 y el alto con los estratos 5 y 6.

** Realizado por un médico y que haya modificado la dieta o el consumo de alcohol en el último año. 
Tabla 5. Razones ajustadas de prevalencia (RP) para el consumo de alcohol en adultos de 18 a 60 años en Bucaramanga, Colombia.

\begin{tabular}{|c|c|c|}
\hline \multirow[b]{2}{*}{ Variable } & \multicolumn{2}{|c|}{ RP (95\% IC)* } \\
\hline & Modelo A & Modelo B \\
\hline \multicolumn{3}{|c|}{ Desinhibición y sentimientos de poder (puntaje) } \\
\hline$<=2$ & 1,00 & 1,00 \\
\hline$>2$ & $1,29(1,03,1,63)^{* * *}$ & $1,33(1,06,1,67)^{* * *}$ \\
\hline \multicolumn{3}{|c|}{ Edad de inicio en el consumo (años) } \\
\hline$>16$ & 1,00 & 1,00 \\
\hline$<=16$ & $1,14(1,07,1,21)^{* * * *}$ & $1,15(1,08,1,23) * * * *$ \\
\hline \multicolumn{3}{|c|}{ Incremento de la expresividad y la sexualidad (puntaje) } \\
\hline$<=2$ & 1,00 & \\
\hline$>2$ & 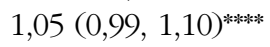 & \\
\hline \multicolumn{3}{|l|}{ Sexo } \\
\hline Mujer & 1,00 & \\
\hline Hombre & $1,01(0,96,1,05)$ & \\
\hline Edad (años) & $1,00(0,99,1,00)$ & \\
\hline
\end{tabular}

$\mathrm{n}=601$. Variable dependiente: Consumo de alcohol; $0=$ No, $1=$ Sí.

*Razones ajustadas de prevalencia e intervalo de confianza del 95\%; Regresión binomial. ${ }^{* *} \mathrm{p}<0,05 .{ }^{* * *} \mathrm{p}$ $<0,001$. ${ }^{* * * * *} \mathrm{p}<0,10$.

ciones diferenciales en las cuatro expectativas estudiadas con la edad, pero sí con la edad de inicio en el consumo. Una posible explicación es que el rango de edad estudiado no permitió identificar esta asociación. Es bien conocido que dependiendo del contexto social donde se realiza el consumo, las experiencias que lo determinan pueden ser incorporadas en edades muy tempranas, entre los cinco y doce años de edad $28-30$. Debido a que la edad en el inicio en el consumo es relativamente alta, es probable que el mecanismo de generación de expectativas en esta población no esté centrado en la experiencia con el consumo.

Tradicionalmente, se ha comunicado que los hombres alcanzan mayores puntuaciones en las EA relacionadas con el desempeño y la conducta sexual $^{20}$. En este estudio no hay evidencia de esto. En esta población, el género no es mediador de las expectativas hacia el consumo de alcohol. Un estudio mexicano que reportó mayores puntuaciones para los hombres en diferentes EA, lo atribuyó a variaciones en los procesos de socialización que se traducen en experimentación diferencial de los efectos del consumo de alcohol ${ }^{31}$.
Es posible argumentar que en esta población a pesar de existir diferentes patrones de crianza dado el género, el contexto social en que se genera el refuerzo hacia el consumo, supera los aspectos particulares de la crianza y que los significados simbólicos de hombres y mujeres asociados con el consumo no son distintos.

La única expectativa asociada al hecho de consumir alcohol "desinhibición y sentimientos de poder", hace parte del contexto social y del comportamiento grupal. Las otras tres no asociadas hacen parte del contexto individual y son predictoras del consumo problemático ${ }^{18,20}$. Es necesario determinar a través de futuros estudios si las expectativas "incremento de la expresividad y la sexualidad", "disminución de la tensión física" y "disminución de la tensión psicológica", se relacionan con el consumo problemático y la dependencia.

En esta población, para la práctica de la salud pública y la generación de la política pública, es necesario identificar y profundizar en otros muchos aspectos, tales como: a) el contexto social que refuerza el consumo, b) la relación de las EA con el consumo problemático y el alcoholismo, c) el 
comportamiento de las EA dependiendo de la cantidad de alcohol consumida, aspecto que podría facilitarse dado el hecho de que para esta población se han diseñado y validado instrumentos para cuantificar el consumo ${ }^{17}$, d) la historia familiar de consumo de alcohol, e) las motivaciones positivas y negativas para consumir alcohol $\mathrm{y}, \mathrm{f}$ ) las conductas de riesgo y la exploración de sensaciones nuevas ${ }^{31}$.

La principal ventaja al usar el AEQ-III en el estudio de las EA, es que debido a su origen en la psicometría, sus resultados pueden tener el carácter de predictores ${ }^{20}$. A pesar de que el diseño de este estudio fue cross-seccional, debido a que las expectativas empiezan a elaborarse en edades tempranas $\mathrm{y}$ antes de consumir alcohol, el modelo binomial presentado podría ser consistente con un proceso causal. Sin embargo, no debe pasarse por alto, que las expectativas y el consumo de alcohol tiene efecto bidireccional y están altamente correlacionadas ${ }^{20}$.

Es importante resaltar que ni la escolaridad o el nivel socioeconómico se asociaron con las EA.

\section{REFERENCIAS}

1. Suter PM. Alcohol: su participación en la salud y la nutrición. En: Bowman AB, Russell RM (eds.). Conocimientos actuales, sobre nutrición. $8^{\mathrm{a}}$ ed. Publicación científica y técnica № 592. Washington, DC: Organización Panamericana de la Salud, 2003; 543-54.

2. Torres GY. Alcohol: Prevalencia de consumo y dependencia en Colombia. Rev Med CES1999; 12: 1-9.

3. Ready KS, Katan MB. Diet, nutrition and the prevention of hypertension and cardiovascular disease. Public Health Nutr 2004; 7: 167-86.

4. Gronbaek M, Becker U, Johansen D, Tonnesen H, Jensen G, Sorensen TI. Population based cohort study of the association between alcohol intake and cancer of the upper digestive tract. BMJ 1998; 317: 844-8.

5. Ministerio de la Protección Social, Fundación FeS Social. Estudio Nacional de Salud Mental Colombia 2003. Bogotá: Ministerio de la Protección Social, 2005.

6. Presidencia de la República. Ministerio de Salud. Programa Rumbos. Estudio Nacional sobre Uso y Abuso de Alcohol y Consumo de Sustancias
Un estudio previo que determinó el patrón de consumo y cuantificó la ingesta de alcohol, había advertido sobre el fracaso de la escuela como regulador social frente a este tema ${ }^{16}$.

En conclusión, a través del estudio de las EA derivadas del AEQ-III, establecimos que el hecho de consumir alcohol está más asociado con el contexto social y la interacción grupal, que con el comportamiento individual. Contrario a la evidencia de otros estudios, en esta población, ni la edad o el género son mediadores de las EA. Además, es muy probable que las EA en esta población se generen en edades muy tempranas. Retardar la edad de inicio en el consumo de alcohol tanto como sea posible y reforzar los elementos positivos asociados con la identidad individual, de grupo, en las relaciones de poder y el liderazgo, deben ser objetivos de futuras intervenciones en escolares y adolescentes para disminuir la prevalencia de consumo de alcohol.

Psicoactivas. Bogotá: Ministerio de Salud, 2000.

7. Luneta P, Penttila A, Sarna S. Water traffic accidents, drowning and alcohol in Finland, 19691995. Inter J Epidemiol 1998; 27: 1038-43.

8. Murphy GE, Wetzel RD, Robins E, McEvoy L. Multiple risk factors predict suicide in alcoholism. Arch Gen Psychiat 1992; 49: 459.

9. Mora J, Natera G. Expectativas, consumo de alcohol y problemas asociados en estudiantes universitarios de la ciudad de México. Salud Pública Mex 2001; 43: 89-96.

10. Miller WR, Rollnick S. Motivational interviewing: Preparing people for change, 2nd Edition, New York: Guilford Press, 2002: 33-42.

11. Rubak S, Sandbaek A, Lauritzen T, Christensen B. Motivational interviewing: a systematic review and meta-analysis. Br J Gen Pract 2005; 55: 305-12.

12. Jhon U, Veltrup C, Driessen M, Wetterling T, Dilurng H. Motivational intervention: An individual counselling vs a group treatment approach for alcohol-dependent in patients. Alcohol alcohol 2003; 38: 263-9.

13. Longabaugh R, Zweben A, Locastro JS, Miller WR. Origins, issues and options in the development of the combined behavioral intervention. J Stud 
Alcohol 2005; Suppl (15): 179-87; discussion 168-9.

14. Fleming A. Alcohol: The delightful poison. A history. $2^{\text {nd }}$ New York: Delacorte Press, 1976.

15. American Psychiatryc Association. Diagnostic and statistical manual of mental disorders. $4^{\text {th }}$ ed. Washington, DC: American Psychiatric Association, 1994.

16. Herrán OF, Ardila MF. Consumo de alcohol, riesgo de alcoholismo y alcoholismo en Bucaramanga, Colombia, 2002-2003. Colombia Med 2005; 36:158-67.

17. Herrán OF, Ardila MF. Validity and Reproducibility of two Semi-Quantitative Alcohol Frequency Questionnaires for Colombian Population. Public Health Nutr 2006; 9: 763-70.

18. Mora-Ríos J, Natera G, Villatorio J, Villalvazo R. Validez factorial del cuestionario de expectativas hacia el alcohol (AEQ-III) en estudiantes universitarios. Psicología Conductual 2000; 8: 319-28.

19. Brown SA, Goldman MS, Inn A, Anderson LR. Expectactions of reinforcement from alcohol their domain and relation to drinking patterns. J Consult Clin Psychol 1980; 48: 419-26.

20. Goldman MS, Del Boca FK, Darles J. Alcohol expectancy theory: The application of cognitive neuroscience. En: Leonard KE, Blane HT (Editors). Psychological theories of drinking and alcoholism. Second Edition. New York: Guilford Press, 1999: 203-46.

21. StataCorp. 2005. Stata Statistical Software: Release 9.1. College Station, TX: StataCorp LP.

22. Brown SA, Christiansen BA, Goldman MS. The alcohol expectancy questionnaire: an instrument for the assessment of adolescent and adult alcohol expectancies. J Stud Alcohol 1987; 48: 483-91.

23. Herrán OF, Ardila MF. Validez factorial del cuestionario de expectativas hacia el consumo de alcohol (AEQ-III), en adultos de Bucaramanga, Colombia. Rev Méd Chile 2007; 135: 488-95.

24. Kleinbaum DG, Kupper LL, Muller KE. Applied regression analysis and other multivariable methods. 2nd ed. Boston: PWS-Kent Publishing Company, 1988, pp. 314-40.

25 . Estimation of means, totals, ratios, and proportions for survey data. Stata Technical Bulletin 31: 623. Reprinted in Stata Technical Bulletinns Reprints 1996: 6: 213-35.

26. Levy SP, Lemeshow S. Sampling of populations: Methods and applications. 3th ed. New York: Jhon Wiley \& Sons, 1999: 365-90.

27. PROFAMILIA. Encuesta Nacional de Demografía y Salud. Resumen de prensa, 2005. Bogotá, Colombia: PROFAMILIA, 2005.

28. Crichlow LB. Belief about the effects of alcohol expectancy scales. J Stud Alcohol 1987; 48: 467-75.

29. Brown S. Context of drinking and reinforcement from alcohol: Alcoholic patterns. Addict Behav 1985;10:191-95.

30. Miller PM, Smith GT, Goldman MS. Emergence of alcohol expectancies in Chilhood: A posible critical period. J Stud Alcohol 1992; 51: 343-9.

31. Cooper LM, Rusell M, Skinner JB, Frone Mr, Mudar P. Stress and alcohol use: Moderating effects of gender, coping and alcohol expectancies. J Abnorm Psychol 1992; 101: 139-52. 